\title{
Low- and high-frequency noise from coherent two-level systems
}

\author{
Alexander Shnirman ${ }^{1}$, Gerd Schön ${ }^{1}$, Ivar Martin $^{2}$, and Yuriy Makhlin ${ }^{3}$ \\ 1 Institut für Theoretische Festkörperphysik Universität Karlsruhe, D-76128 Karlsruhe, Germany \\ ${ }^{2}$ Theoretical Division, Los Alamos National Laboratory, Los Alamos, NM 87545, USA \\ ${ }^{3}$ Landau Institute for Theoretical Physics, Kosygin st. 2, 119334 Moscow, Russia
}

(Dated: February 2, 2008)

\begin{abstract}
Recent experiments indicate a connection between the low- and high-frequency noise affecting superconducting quantum systems. We explore the possibilities that both noises can be produced by one ensemble of microscopic modes, made up, e.g., by sufficiently coherent two-level systems (TLS). This implies a relation between the noise power in different frequency domains, which depends on the distribution of the parameters of the TLSs. We show that a distribution, natural for tunneling TLSs, with a log-uniform distribution in the tunnel splitting and linear distribution in the bias, accounts for experimental observations.
\end{abstract}

Recent activities and progress with quantum information systems rely on the control of decoherence processes and at the same time provide novel tools to study their mechanisms. Experiments with superconducting qubits revealed the presence of spurious quantum two-level systems [1] with strong effects on the high-frequency $(\sim 10$ $\mathrm{GHz})$ qubit dynamics. Other experiments [2] suggested a connection between the strengths of the Ohmic highfrequency noise, responsible for the relaxation of the qubit ( $T_{1}$ decay), and the low-frequency $1 / f$ noise, which dominates the dephasing ( $T_{2}$ decay). The noise power spectra, extrapolated from the low- and high-frequency sides, cross at $\omega$ of order $T$. This is also compatible with the $T^{2}$ dependence of the low-frequency part, observed earlier for the $1 / f$ noise in Josephson devices [3, 4]. Much clearer evidence for the $T^{2}$ behavior was obtained recently [5, 6].

In this letter we point out that a set of coherent twolevel systems (or, in fact, arbitrary quantum systems with discrete spectrum) produces both high- and lowfrequency noise with strengths that are naturally related. We show that for a realistic distribution of parameters tunnel TLSs (TTLS) produce noise with experimentally detected features: the $1 / f$ behavior at low frequencies, the Ohmic $(\propto \omega)$ high-frequency noise, and the $T^{2}$ temperature dependence of the integrated weight of the lowfrequency noise. This implies that the $1 / f$ and Ohmic asymptotes cross at $\omega \sim T$ as was indeed observed in Ref. [2] at one value of $T$. The distribution is log-uniform in the tunnel splitting and linear in the bias. Microscopically, this distribution may describe double traps or "Andreev fluctuators" considered recently by Faoro et al. 7] in their study of the relaxation ( $T_{1}$ decay) of Josephson qubits due to the high-frequency noise. Our results are obtained for environments with a large number of TLSs which are weakly coupled to the qubit. A strong coupling between a TLS and a qubit can lead to resonances [1, 2].

Ensembles of TLSs were discussed extensively in the literature. On one hand, they produce a natural model of $1 / f$ noise, as a result of incoherent random transitions [8], and there is substantial experimental evidence that the low-frequency $1 / f$ noise in single-electron devices may be produced by TLSs [9, 10]. In solid-state qubits, e.g., Josephson qubits, the pure dephasing is dominated by this noise [11, 12, 13]. On the other hand, ensembles of coherent TTLSs were suggested to explain lowtemperature properties of glasses [14, 15]. Both "transverse" and "longitudinal" couplings, defined below, were discussed in relation to various physical phenomena. A transverse coupling of phonons or electrons to the TLSs is responsible for the absorption and emission of energy. It was invoked in the discussions of, e.g., the phonon attenuation [16] and of the low-temperature dephasing in disordered metals [17]. On the other hand a longitudinal coupling was found to be responsible, e.g., for the conductance fluctuations [18, 19, 20]. We suggest that in nanocircuits, e.g., solid-state qubits, both types of couplings play an important part and produce noise with related properties in various frequency ranges.

As a model we consider a set of coherent two-level systems described by the Pauli matrices $\sigma_{p, j}$, where $p=x, y, z$ and $j$ is the index of a particular TLS. We write the Hamiltonian of the set in the basis defined by their contributions to the relevant fluctuating quantity (cf. Eq. (2) below);

$$
H_{\mathrm{TLS}}=\sum_{j}\left[-\frac{1}{2}\left(\varepsilon_{j} \sigma_{z, j}+\Delta_{j} \sigma_{x, j}\right)+H_{\mathrm{diss}, j}\right] .
$$

Here, in the language of TTLSs, $\varepsilon_{j}$ are the bias energies and $\Delta_{j}$ are the tunnel amplitudes between two states. Each TLS with label $j$ is subject to dissipation due to its own bath with Hamiltonian $H_{\text {diss }, j}$. We do not specify $H_{\text {diss }, j}$, but only assume that it produces the usual relaxation $\left(T_{1}\right)$ and dephasing $\left(T_{2}\right)$ processes. We assume that all the TLSs are under-damped, with $\Gamma_{1, j} \equiv T_{1, j}^{-1} \ll E_{j}$ and $\Gamma_{2, j} \equiv T_{2, j}^{-1} \ll E_{j}$. Here $E_{j} \equiv \sqrt{\epsilon_{j}^{2}+\Delta_{j}^{2}}$ is the energy splitting.

Each TLS in the ensemble contributes to fluctuations of a physical quantity $X$, e.g., the gate charge, which affect an experimentally accessible system and thus may be detected. A qubit may serve as a convenient noise 
detector 21, 22]. E.g., in the recent experiment of Ref. [2] qubits were used to investigate the properties of their environment. We choose

$$
X \equiv \sum_{j} v_{j} \sigma_{z, j},
$$

where $v_{j}$ are the coupling constants and $\sigma_{z, j}= \pm 1$ correspond to the two states differing, e.g., by the value of the dipole moment. The interaction of the qubit with the TLSs is often described via a linear in $X$ coupling to a variable $O_{\text {qubit }}$ of the qubit, i.e., $H_{\text {int }}(X) \propto X O_{\text {qubit }}$.

Our goal in the following is to investigate the noise properties of $X$, that is we need to evaluate the (unsymmetrized) correlator

$$
C_{X}(\omega) \equiv \int d t\left\{\langle X(t) X(0)\rangle-\langle X\rangle^{2}\right\} e^{i \omega t} .
$$

For independent TLSs the noise is a sum of individual contributions, $C_{X}=\sum_{j} v_{j}^{2} C_{j}$, where

$$
C_{j}(\omega) \equiv \int d t\left\{\left\langle\sigma_{z, j}(t) \sigma_{z, j}(0)\right\rangle-\left\langle\sigma_{z, j}\right\rangle^{2}\right\} e^{i \omega t} .
$$

To obtain $C_{j}$ we first transform to the eigenbasis of the TLS. This gives

$$
H_{\mathrm{TLS}}=\sum_{j}\left\{-\frac{1}{2} E_{j} \rho_{z, j}+H_{\mathrm{diss}, j}\right\},
$$

and

$$
X=\sum_{j} v_{j}\left(\cos \theta_{j} \rho_{z, j}-\sin \theta_{j} \rho_{x, j}\right),
$$

where $\tan \theta_{j} \equiv \Delta_{j} / \epsilon_{j}$. The first term of (6) produces the longitudinal coupling (mentioned above) while the second term produces the transverse one. Using the BlochRedfield theory [23, 24] we find readily

$$
\begin{aligned}
C_{j}(\omega) & \approx \cos ^{2} \theta_{j}\left[1-\left\langle\rho_{z, j}\right\rangle^{2}\right] \frac{2 \Gamma_{1, j}}{\Gamma_{1, j}^{2}+\omega^{2}} \\
& +\sin ^{2} \theta_{j}\left[\frac{1+\left\langle\rho_{z, j}\right\rangle}{2}\right] \frac{2 \Gamma_{2, j}}{\Gamma_{2, j}^{2}+\left(\omega-E_{j}\right)^{2}} \\
& +\sin ^{2} \theta_{j}\left[\frac{1-\left\langle\rho_{z, j}\right\rangle}{2}\right] \frac{2 \Gamma_{2, j}}{\Gamma_{2, j}^{2}+\left(\omega+E_{j}\right)^{2}}
\end{aligned}
$$

In thermal equilibrium $\left\langle\rho_{z, j}\right\rangle=\tanh \left(E_{j} / 2 T\right)$. The first term, due to the longitudinal part of the coupling, describes random telegraph noise of a thermally excited TLS. We have assumed $\Gamma_{1, j} \ll T$, so that this term is symmetric (classical). The second term is due to the transverse coupling and describes absorption by the TLS, while the third term describes the transitions of the TLS with emission. We observe that TLSs with $E_{j} \gg T$ contribute to $C_{X}$ only at (positive) $\omega=E_{j}$. Indeed their contribution at $\omega=0$ is suppressed by the thermal factor $1-\left\langle\rho_{z, j}\right\rangle^{2}=1-\tanh ^{2}\left(E_{j} / 2 T\right)$. Also the negative frequency (emission) contribution at $\omega=-E_{j}$ is suppressed. These high-energy TLSs remain always in their ground state. Only the TLSs with $E_{j}<T$ are thermally excited, performing real random transitions between their two eigenstates, and contribute both at $\omega= \pm E_{j}$ and at $\omega=0$. Such a multi-peaked structure of $C_{j}(\omega)$ was recently discussed in various contexts, e.g., in Refs. 25, 26, 27]. Note that the separation of the terms in Eq. (17) into low- and high-frequency noise is meaningful provided the typical width $\Gamma_{1, j}$ of the low- $\omega$ Lorentzians is lower than the high frequencies of interest, which are defined, e.g., by the qubit's level splitting or temperature.

For a dense distribution of the parameters $\epsilon, \Delta$, and $v$ we can evaluate the low- and high-frequency noise. For positive high frequencies, $\omega \gg T$, we obtain

$$
\begin{aligned}
& C_{X}(\omega) \approx \sum_{j} v_{j}^{2} \sin ^{2} \theta_{j} \frac{2 \Gamma_{2, j}}{\Gamma_{2, j}^{2}+\left(\omega-E_{j}\right)^{2}} \\
& \approx N \int d \epsilon d \Delta d v P(\epsilon, \Delta, v) v^{2} \sin ^{2} \theta \cdot 2 \pi \delta(\omega-E),
\end{aligned}
$$

where $N$ is the number of fluctuators, $P(\epsilon, \Delta, v)$ is the distribution function normalized to $1, E \equiv \sqrt{\epsilon^{2}+\Delta^{2}}$, and $\tan \theta=\Delta / \epsilon$. Without loss of generality we take $\epsilon \geq 0$ and $\Delta \geq 0$. At negative high frequencies $(\omega<$ 0 and $|\omega|>T)$ the correlator $C_{X}(\omega)$ is exponentially suppressed.

On the other hand, the total weight of the lowfrequency (up to $\omega \gtrsim \Gamma_{1}$ ) noise follows from the first term of (7). Each Lorentzian contributes 1. Thus we obtain

$$
\begin{aligned}
& \int_{\text {low freq. }} \frac{d \omega}{2 \pi} C_{X}(\omega) \\
& \approx \int_{\text {low freq. }} \frac{d \omega}{2 \pi} \sum_{j} v_{j}^{2} \cos ^{2} \theta_{j}\left[1-\left\langle\rho_{z, j}\right\rangle^{2}\right] \frac{2 \Gamma_{1, j}}{\Gamma_{1, j}^{2}+\omega^{2}} \\
& \approx N \int d \epsilon d \Delta d v P(\epsilon, \Delta, v) v^{2} \cos ^{2} \theta \frac{1}{\cosh ^{2} \frac{E}{2 T}} .
\end{aligned}
$$

Here we could disregard the contribution of the last two terms for $E_{j} \sim \Gamma_{1, j}$. Equations (8) and (9) provide the general framework for further discussion.

Next we investigate possible distributions for the parameters $\epsilon, \Delta$, and $v$. We consider a $\log$-uniform distribution of tunnel splittings $\Delta$, with density $P_{\Delta}(\Delta) \propto 1 / \Delta$ in a range $\left[\Delta_{\min }, \Delta_{\max }\right]$. This distribution is natural for TTLSs as $\Delta$ is an exponential function of an almost uniformly distributed parameter, e.g., tunnel barrier height [15]. It is also well known to provide for 
the $1 / f$ behavior of the low-frequency noise [8]: the relaxation rates are, then, also distributed log-uniformly, $P_{\Gamma_{1}}\left(\Gamma_{1}\right) \propto 1 / \Gamma_{1}$, and a sum of many Lorentzians of width $\Gamma_{1}$ centered at $\omega=0$ adds up to the $1 / f$ noise. We further assume that the distribution of $v$ is uncorrelated with $\varepsilon$ and $\Delta$.

First, we assume that the temperature is lower than $\Delta_{\max }, T<\Delta_{\max }$. For the high-frequency part, $T<\omega<$ $\Delta_{\max }$, taking the integral over $\Delta$ in Eq. (8), we find that

$$
C_{X}(\omega) \propto \frac{1}{\omega} \int_{0}^{\omega} P_{\varepsilon}(\varepsilon) d \varepsilon .
$$

This is consistent with an Ohmic behavior $C_{X} \propto \omega$ only for the linear density $P_{\varepsilon}(\varepsilon) \propto \varepsilon$.

Importantly, this distribution $P(\varepsilon, \Delta) \propto \varepsilon / \Delta$ leads at the same time to the $T^{2} \ln \left(T / \Delta_{\text {min }}\right)$ behavior of the lowfrequency weight (9), consistent with experimental observations $[3,4,5,6]$. If the low-frequency noise has a $1 / f$ dependence, the two parts of the spectrum would cross around $\omega \sim T$ [2].

A remark is in order concerning this crossing. It is not guaranteed that the spectrum has a $1 / f$ dependence up to $\omega \sim T$. Rather the high-frequency cutoff of the lowfrequency $1 / f$ noise is given by the maximum relaxation rate of the TLSs, $\Gamma_{1, \max } \ll T$, as we assumed. Then the extrapolations of the low-frequency $1 / f$ and highfrequency Ohmic spectra cross at this $\omega \sim T$.

To fix the coefficients, we introduce the normalization constant $A$, so that $P(\epsilon, \Delta)=A \epsilon / \Delta$. Then, at high positive frequencies, $T<\omega<\Delta_{\max }$, we obtain

$$
C_{X}(\omega) \approx \pi\left\langle v^{2}\right\rangle N A \omega
$$

For the total weight of the low-frequency noise we obtain

$$
\int_{\text {low freq. }} \frac{d \omega}{2 \pi} C_{X}(\omega) \approx 4 \ln (2)\left\langle v^{2}\right\rangle N A T^{2}\left[\ln \frac{T}{\Delta_{\min }}\right]
$$

Thus we obtain a numerical factor which determines precisely the point of crossing of the two spectra.

In the opposite limit, $T \gg \Delta_{\max }$, the high/frequency noise depends on the detailed shape of the cutoff of $P_{\Delta}(\Delta)$ at $\Delta_{\max }$. As an example, for a hard cutoff the Ohmic spectral density implies that $P_{\varepsilon} \propto \varepsilon^{3}$, and the lowfrequency weight scales with $T^{4}$. For a $1 / f$ low-frequency behavior, the spectra would cross at $\omega \sim T^{2} / \Delta_{\max } \gg T$, in disagreement with the result of Ref. [2].

Interestingly, the linear $\omega$ dependence at high frequencies and the $T^{2}$ dependence of the low-frequency noise can be obtained from a whole class of distributions, e.g., for $P(\epsilon, \Delta)=f(\epsilon / \Delta)$, with arbitrary, not too divergent (as a function of $\theta$ ) function $f$. Presented as a function of energy $E$ and angle $\theta$, it becomes (we have used the Jacobian $d \epsilon d \Delta \rightarrow E d E d \theta)$

$$
P(E, \theta)=E f(\cot \theta),
$$

i.e., it is linear in $E$. This linearity ensures both the linear $\omega$ dependence at high frequencies and the $T^{2}$ dependence of the integrated weight of the low-frequency noise.

In particular one can take $P(\epsilon, \Delta) \propto(\epsilon / \Delta)^{s}$ with any exponent $s$ satisfying $-1 \leq s \leq 1$, and with both $\Delta_{\max }$ and $\epsilon_{\max }$ higher than the relevant frequency $\omega$. This includes a uniform distribution of both $\Delta$ and $\epsilon$ at all relevant energies, i.e., $s=0$. For ensembles with $-1<s<1$, including the uniform distribution with $s=0$, high- and low-frequency noise is created by the same fluctuators. On the other hand, $s=1$ is the limiting case in which the low-frequency noise is dominated by the fluctuators with $\theta \ll 1$, while the high-frequency noise by all other fluctuators. Yet, even in this case the strengths of the high- and low-frequency parts of the spectrum are related.

For the uniform distribution $(s=0)$ we obtain different numerical coefficients. We introduce an experimentally accessible constant $\alpha$, such that $C_{X}(\omega \gg T)=2 \pi \alpha \omega$. Then, for $s=1(P \propto \epsilon / \Delta)$ we obtain from Eqs. (11) and (12) that the total weight of the low-frequency noise is given by $\int_{\text {l.f. }} \frac{d \omega}{2 \pi} C_{X}(\omega) \approx 8 \ln (2) \alpha T^{2} \ln \left(T / \Delta_{\text {min }}\right)$. On the other hand, for the uniform distribution, $s=0$, we obtain $\int_{\text {l.f. }} \frac{d \omega}{2 \pi} C_{X}(\omega) \approx 4 \ln (2) \alpha T^{2}$.

We would like to emphasize that the relation between low- and high-frequency noise is more general, i.e., it is not unique to an ensemble of two-level systems. Consider an ensemble of many-level systems with levels $|n\rangle$ and energies $E_{n}$ such that the coupling is via an observable which has both transverse and longitudinal components. By a transverse component we mean the part constructed with operators $|n\rangle\langle m|$, where $n \neq m$, while the longitudinal component is built from the projectors $|n\rangle\langle n|$. If the system is under-damped, that is, if the absorption and emission lines are well defined, the correlator of such an observable will have (Lorentzian-like) contributions at $\omega=E_{n}-E_{m}$ as well as at $\omega=0$. As an example we could consider an ensemble of an-harmonic oscillators with $X=\sum_{j} v_{j} x_{j}$, where $x_{j}$ are the oscillator's coordinates. Due to the anharmonicity $x_{j}$ acquires a longitudinal component, in addition to the usual transverse one. Thus a relation between the low- and high-frequency noise would emerge naturally with details depending on the ensemble statistics.

It is useful to relate our phenomenological results to the recent work of Faoro et al. [7], where they considered physical models of the fluctuators, which could couple to and relax qubits. They considered three models: (I) a single electron trap in tunnel contact with a metallic gate, (II) a single electron occupying a double trap, and (III) a double trap that can absorb/emit a Cooper pair from the qubit or a superconducting gate. In all models a uniform distribution of the trap energy levels was assumed. One, then, can show that the distribution for the two-level systems corresponding to the models II and III are linear in the energy level splitting, $P(\epsilon) \propto \epsilon$. Since 
the switching in these models is tunneling dominated, we find that $P(\Delta) \propto 1 / \Delta$. Therefore, both models II and III are characterized by distribution $P(\epsilon, \Delta)=A \epsilon / \Delta$, described above, and hence can naturally account for the experimentally observed low- and high-frequency noises. In contrast, in the model of uniformly distributed singleelectron traps (model I), we find that for small tunnel rates, the high-frequency noise is inversely proportional to frequency rather than Ohmic [7].

In this letter we did not address the question of the statistics of the low-frequency noise, nor the associated problem of a particular decay law of the dephasing process. These statistics will depend on the distribution of the coupling strengths $v_{j}$. For certain distributions the individual strongly coupled fluctuators may be important 28, 29], and the statistics is non-Gaussian. For ensembles of many weakly coupled fluctuators Gaussian statistics emerges [11, 30].

To conclude, we have shown that an ensemble of coherent two-level systems with the distribution function, $P(\epsilon, \Delta) \propto \epsilon / \Delta$, produces Ohmic high-frequency noise and, at the same time, $1 / f$ low-frequency noise with strength which grows with temperature as $T^{2}$. The two branches of the noise power cross at $\omega \sim T$ in accordance with the experimental observation [2]. A relation between low- and high-frequency parts of the spectrum is a general property of ensembles of coherent systems with discrete energy levels.

We are grateful to Yu. A. Pashkin for communicating the experimental results of Ref. 2] prior to publication. We thank J. Clarke and O. Astafiev for stimulating discussions and useful comments. The work is part of the CFN of the DFG and of the EU IST Project SQUBIT. This work was supported by the U.S. DoE. YM acknowledges support from the Dynasty Foundation.

[1] R. W. Simmonds, K. M. Lang, D. A. Hite, S. Nam, D. P. Pappas, and J. M. Martinis, Phys. Rev. Lett. 93, 077003 (2004).

[2] O. Astafiev, Yu. A. Pashkin, Y. Nakamura, T. Yamamoto, and J. S. Tsai, Phys. Rev. Lett. 93, 267007 (2004).

[3] F. C. Wellstood, PhD thesis, University of California, Berkeley (1988).

[4] M. Kenyon, C. J. Lobb, and F. C. Wellstood, J. Appl. Phys. 88, 6536 (2000).
[5] O. Astafiev et al., private communication (2004).

[6] F. C. Wellstood, C. Urbina, and J. Clarke, Appl. Phys. Lett. 85, 5296 (2004).

[7] L. Faoro, J. Bergli, B. A. Altshuler, and Y. M. Galperin, cond-mat/0411425 (2004).

[8] P. Dutta and P. M. Horn, Rev. Mod. Phys. 53, 497 (1981).

[9] G. Zimmerli, T. M. Eiles, R. L. Kautz, and J. M. Martinis, Appl. Phys. Lett. 61, 237 (1992).

[10] A. B. Zorin, F.-J. Ahlers, J. Niemeyer, T. Weimann, H. Wolf, V. A. Krupenin, and S. V. Lotkhov, Phys. Rev. B 53, 13682 (1996).

[11] Y. Nakamura, Yu. A. Pashkin, T. Yamamoto, and J. S. Tsai, Phys. Rev. Lett. 88, 047901 (2002).

[12] D. Vion, A. Aassime, A. Cottet, P. Joyez, H. Pothier, C. Urbina, D. Esteve, and M. H. Devoret, Science 296, 886 (2002).

[13] D. J. VanHarlingen, T. L. Robertson, B. L. T. Plourde, P. A. Reichardt, T. A. Crane, and J. Clarke, Phys. Rev. B 70, 064517 (2004).

[14] P. W. Anderson, B. I. Halperin, and C. M. Varma, Phylos. Mag. 25, 1 (1972).

[15] W. A. Phillips, J. Low. Temp. Phys. 7, 351 (1972).

[16] J. L. Black, in Glassy metals, edited by H.-J. Güntherodt and H. Beck (Springer-Verlag, Berlin, 1981).

[17] Y. Imry, H. Fukuyama, and P. Schwab, Europhys. Lett. 47, 608 (1999).

[18] S. M. Kogan and K. E. Nagaev, Solid State Comm. 49, 387 (1984).

[19] A. Ludviksson, R. Kree, and A. Schmid, Phys. Rev. Lett. 52, 950 (1984).

[20] S. Feng, P. A. Lee, and A. D. Stone, Phys. Rev. Lett. 56, 1960 (1986).

[21] R. Aguado and L. P. Kouwenhoven, Phys. Rev. Lett. 84, 1986 (2000).

[22] R. J. Schoelkopf, A. A. Clerk, S. M. Girvin, K. W. Lehnert, and M. H. Devoret, Proceedings of NATO ARW, Delft, June 2-4, 2002, cond-mat/0210247. (2002).

[23] F. Bloch, Phys. Rev. 105, 1206 (1957).

[24] A. G. Redfield, IBM J. Res. Dev. 1, 19 (1957).

[25] A. N. Korotkov and D. V. Averin, Phys. Rev. B 64, 165310 (2001).

[26] A. Shnirman, D. Mozyrsky, and I. Martin, Europhys. Lett. 67, 840 (2004).

[27] Y. M. Galperin, V. I. Kozub, and V. M. Vinokur, Phys. Rev. B 70, 033405 (2004).

[28] E. Paladino, L. Faoro, G. Falci, and R. Fazio, Phys. Rev. Lett. 88, 228304 (2002).

[29] Y. M. Galperin, B. L. Altshuler, and D. V. Shantsev, cond-mat/0312490 (2003).

[30] A. Shnirman, Yu. Makhlin, and G. Schön, Physica Scripta T102, 147 (2002). 